\title{
A NOTE ON THE BOREL FORMULA
}

\author{
RONALD M. DOTZEL
}

\begin{abstract}
A new proof of the Borel formula is obtained for $G=\left(Z_{p}\right)^{r}$ actions on spaces $X$ having $H_{i}\left(X ; Z_{p}\right)=0, i \neq n$ (some $n$ ) and $H_{n}\left(X ; Z_{p}\right)=Z_{p} \oplus$ Free $Z_{p} G$ module. Each $X^{H}$ must be a $Z_{p}$-homology $n(H)$-sphere and then $n-n(G)=$ $\Sigma(n(H)-(G))$, sum running over corank 1 subgroups. A discussion of examples follows.
\end{abstract}

1. Introduction. If an elementary abelian $p$-group $G=\left(Z_{p}\right)^{r}$ acts on a $Z_{p}$-homology $n$-sphere, then Smith theory [3, Chapter 3] says that the fixed point set of a subgroup $H$ of $G$ is a $Z_{p}$-homology $n(H)$-sphere. If $p$ is odd $n-n(H)$ must be even for all $H$. The Borel formula [2, p. 175] states that, moreover, $n-n(G)=$ $\Sigma(n(H)-n(G))$ where the sum runs over all subgroups $H$ of $G$ such that $G / H=Z_{p}$ (i.e. $H$ has corank 1 ).

In [4] the following converse result was established.

TheOREM A. Let $X$ be a finite $C W$ complex with a cellular action of $G=\left(Z_{p}\right)^{r}$, such that each $X^{H}(H \neq 0)$ is a $Z_{p}$-homology $n(H)$-sphere. Suppose, moreover, that there exists an $n$ such that $\tilde{H}_{i}\left(X ; Z_{p}\right)=0, i \neq n$, and so that if $p$ is odd, $n-n(G)$ is even. Also assume the Borel formula holds for this action (and $n)$. Then $H_{n}\left(X ; Z_{p}\right)=$ $Z_{p} \oplus F$ where $F$ is $Z_{p} G$ free.

This result was established by constructing a linear action of $G$ on $S^{\boldsymbol{n}}$ and an equivariant map $\phi: X \rightarrow S^{n}$ which induces $Z_{p}$-homology isomorphisms $\phi^{H}: X^{H} \rightarrow$ $\left(S^{n}\right)^{H}, H \neq 0$, and a $Z_{p}$-homology epimorphism when $H=0$.

In the present paper we establish the converse to Theorem $\mathrm{A}$, which provides an extension of the Borel formula. I am indebted to Professor Glen Bredon, who first suggested the approach I used here, and to Professor Gary C. Hamrick for many helpful conversations.

2. We begin with

Proposition 1. Let $G=\left(Z_{p}\right)^{r}(r>1)$ act cellularly on $X$, a finite $C W$ complex such that each $X^{H}(H \neq 0)$ is a $Z_{p}$-homology $n(H)$-sphere. Suppose there is an integer $n$ so that $\tilde{H}_{i}\left(X ; Z_{p}\right)=0, i \neq n$, and $H_{n}\left(X ; Z_{p}\right)=Z_{p} \oplus F$, where $F$ is free over $Z_{p} G$. Then the Borel formula holds, i.e.,

$$
n-n(G)=\sum(n(H)-n(G)),
$$

sum extending over all corank 1 subgroups $H$ in $G$.

Received by the editors August 14, 1978 and, in revised form, December 4, 1978.

AMS (MOS) subject classifications (1970). Primary 55C35; Secondary 55B25.

(C) 1980 American Mathematical Society 0002-9939/80/0000-0176/\$02.25 
Proof. Let $k$ be the cellular dimension of $X$. The proof proceeds in roughly two stages. At first we construct inductively a finite $\mathrm{CW}$ complex, $Y_{k-1}$, with the property that $Y_{k-1}$ is $k$-dimensional, $k-1$-connected. $Y_{k-1}$ will also have the property that

$$
\operatorname{Ext}_{Z_{p} G}^{s+(k-n)}\left(H_{n}\left(X ; Z_{p}\right), Z_{p}\right)=\operatorname{Ext}_{Z_{p} G}^{s}\left(H_{k}\left(Y_{k-1} ; Z_{p}\right), Z_{p}\right)
$$

for all $s \geqslant 2$.

On the other hand we define a finite $\mathrm{CW}$ complex $\hat{Y}_{k-1}$ which satisfies the hypothesis of Theorem $\mathrm{A}$ in the introduction, for the integer $m=\Sigma(n(H)-n(G))$ $+n(G)$ (not, at this point, necessarily equal to $n$ ). It will result that

$$
\operatorname{Ext}_{Z_{p} G}^{s+(k-n)}\left(H_{n}\left(X ; Z_{p}\right), Z_{p}\right)=\operatorname{Ext}_{Z_{p} G}^{s+(k-m)}\left(H_{m}\left(\hat{Y}_{k-1} ; Z_{p}\right), Z_{p}\right)
$$

for any $s \geqslant 2$. Since $G$ has nonperiodic group cohomology for $r>1$, it follows that $n=m$, which is what we wanted to show.

To begin with, we will use extensively the fact that whenever $f: M \rightarrow T \oplus P$ is a $Z G$ epimorphism of $Z G$ modules with $M$ free, $P$ projective and $T$ consisting of torsion prime to $p, \operatorname{ker} f$ is $Z G$ projective. This follows from [7, 4.12] once it is clear that $T$ is cohomologically trivial. But multiplication by $|G|$ on $T$ is an automorphism, which induces an automorphism on cohomology. Coupling this with the fact that $H^{*}(G, \cdot)$ has exponent $|G|$ (see Cartan-Eilenberg, Chapter XII, e.g.), it follows that $T$ is cohomologically trivial (this argument was pointed out to me by R. Oliver).

We may assume $X$ is 1 -connected (by suspending). Since $\tilde{H}_{i}(X ; Z)$ is torsion prime to $p$, we may attach free orbits of cells in dimensions $\leqslant n$ to produce the complex $Y$ such that $Y$ is $n-1$-connected and $H_{n}(Y ; Z)=H_{n}(X ; Z) \oplus N, N$ a $Z G$-projective (for a nice exposition of the procedure of "equivariantly attaching cells", see [5, $\$ 1])$. Now add free orbits of $n+1$-cells to kill off $N$. This produces a complex $X_{0}$ with $H_{n}\left(X_{0} ; Z\right)=H_{n}(X ; Z)$ and $H_{n+1}\left(X_{0} ; Z\right)=H_{n+1}(X ; Z) \oplus$ $N_{0}, N_{0}$ a $Z G$-projective. $H_{i}\left(X_{0} ; Z\right)=H_{i}(X ; Z), i>n+1$.

Add free orbits of $n+1$-cells to $X_{0}$ to kill off $H_{n}(X ; Z)$. This creates an $n$-connected, $k$-dimensional complex $Y_{n}$, so that $H_{i}\left(Y_{n}\right)=H_{i}(X), i>n+1$, and

$$
0 \rightarrow H_{n+1}\left(X_{0} ; Z\right) \rightarrow H_{n+1}\left(Y_{n} ; Z\right) \rightarrow \operatorname{Ker} \partial_{n+1} \rightarrow 0
$$

where $0 \rightarrow \operatorname{Ker} \partial_{n+1} \rightarrow H_{n+1}\left(Y_{n}, X_{0} ; Z\right) \rightarrow H_{n}\left(X_{0} ; Z\right) \rightarrow 0$. Now if $k=n+1$, it follows that $H_{n+1}\left(X_{0} ; Z\right)=N_{0}$, since $H_{n+1}\left(X ; Z_{p}\right)=0$. By [7, 3.5], $N_{0} \otimes Z_{p}$ is $Z_{p} G$ free. After tensoring the above sequences with $Z_{p}$, it follows that

$$
\operatorname{Ext}_{Z_{p} G}^{s}\left(H_{n}\left(X ; Z_{p}\right), Z_{p}\right)=\operatorname{Ext}_{Z_{p} G}^{s-1}\left(H_{n+1}\left(Y_{n} ; Z_{p}\right), Z_{p}\right) \text { for any } s \geqslant 2 \text {. }
$$

If $k>n+1$, we show below how to construct $Y_{n+1}$, which is $n+1$-connected and $k$-dimensional, from $Y_{n}$. If need be, the following argument may be repeated until one obtains $Y_{k-1}$.

Add free orbits of $n+2$-cells to $Y_{n}$ to kill off $H_{n+1}\left(X_{0} ; Z\right)$ (see sequence (*)). This produces a complex $Y_{n+1}^{\prime}$ with $H_{n+1}\left(Y_{n+1}^{\prime} ; Z\right)=\operatorname{Ker} \partial_{n+1}$ and $H_{n+2}\left(Y_{n+1}^{\prime} ; Z\right)=H_{n+2}\left(X_{0} ; Z\right) \oplus N_{1}$, where $N_{1}$ is $Z G$-projective. 
Now add free orbits of $n+2$-cells to $Y_{n+1}^{\prime}$ to form $Y_{n+1}$ and to kill off $\operatorname{Ker} \partial_{n+1}$. $Y_{n+1}$ is $n+1$-connected, $k$-dimensional. Further, we have:

$$
H_{n+2}\left(Y_{n+1}, Y_{n+1}^{\prime} ; Z\right) \rightarrow H_{n+1}\left(Y_{n+1}^{\prime}, X_{0} ; Z\right) \stackrel{\partial_{n+1}}{\rightarrow} H_{n}\left(X_{0} ; Z\right) \rightarrow 0
$$

where the left-hand map is the composition

$$
H_{n+2}\left(Y_{n+1}, Y_{n+1}^{\prime} ; Z\right) \rightarrow H_{n+1}\left(Y_{n+1}^{\prime} ; Z\right) \approx \operatorname{Ker} \partial_{n+1} \mapsto H_{n+1}\left(Y_{n+1}^{\prime}, X_{0} ; Z\right) \text {. }
$$

We also have the exact sequence,

$$
\begin{aligned}
0 & \rightarrow H_{n+2}\left(Y_{n+1}^{\prime} ; Z\right) \rightarrow H_{n+2}\left(Y_{n+1} ; Z\right) \\
& \rightarrow H_{n+2}\left(Y_{n+1}, Y_{n+1}^{\prime} ; Z\right) \stackrel{\partial_{n+2}}{\rightarrow} H_{n+1}\left(Y_{n+1}^{\prime} ; Z\right) .
\end{aligned}
$$

Now if $k=n+2$, it follows that $H_{n+2}\left(Y_{n+1}^{\prime} ; Z\right)=N_{1}$, a $Z G$-projective, since $H_{n+2}\left(X ; Z_{p}\right)=0$. Thus $H_{n+2}\left(Y_{n+1}^{\prime} ; Z_{p}\right)$ is $Z_{p} G$ free. As before we have the exact sequences

$$
\begin{aligned}
& 0 \rightarrow H_{n+2}\left(Y_{n+1}^{\prime} ; Z_{p}\right) \rightarrow H_{n+2}\left(Y_{n+1} ; Z_{p}\right) \rightarrow \operatorname{Ker} \partial_{n+2} \otimes Z_{p}, \\
& 0 \rightarrow \operatorname{Ker} \partial_{n+2} \otimes Z_{p} \rightarrow H_{n+2}\left(Y_{n+1}, Y_{n+1}^{\prime} ; Z_{p}\right) \rightarrow H_{n+1}\left(Y_{n+1}^{\prime} ; Z_{p}\right) .
\end{aligned}
$$

Then

$$
\operatorname{Ext}_{Z_{p} G}^{s}\left(H_{n+1}\left(Y_{n+1}^{\prime} ; Z_{p}\right), Z_{p}\right)=\operatorname{Ext}_{Z_{p} G}^{s-1}\left(H_{n+2}\left(Y_{n+1} ; Z_{p}\right), Z_{p}\right)
$$

and

$$
\operatorname{Ext}_{Z_{p} G}^{s}\left(H_{n}\left(X ; Z_{p}\right), Z_{p}\right)=\operatorname{Ext}_{Z_{p} G}^{s-1}\left(H_{n+1}\left(Y_{n+1}^{\prime} ; Z_{p}\right), Z_{p}\right)
$$

since $H_{n+1}\left(Y_{n+1}^{\prime} ; Z_{p}\right)=\operatorname{Ker} \partial_{n+1} \otimes Z_{p}$ and where $s$ is arbitrary, $s \geqslant 2$.

If $k>n+2$, we have the exact sequence

$$
0 \rightarrow H_{n+2}\left(Y_{n+1}^{\prime} ; Z\right) \rightarrow H_{n+2}\left(Y_{n+1} ; Z\right) \rightarrow \operatorname{Ker} \partial_{n+2}
$$

Since $H_{n+2}\left(Y_{n+1}^{\prime} ; Z\right)=H_{n+2}\left(X_{0} ; Z\right) \oplus N_{1}$, we can repeat the procedure above to reach, eventually, $Y_{k-1}$. By adding additional free orbits of cells, if necessary, we may assume $k-m \geqslant 2$.

The same argument as above shows that for any $s \geqslant 2$,

$$
\operatorname{Ext}_{Z_{p} G}^{s+(k-n)}\left(H_{n}\left(X ; Z_{p}\right), Z_{p}\right)=\operatorname{Ext}_{Z_{p} G}^{s}\left(H_{k}\left(Y_{k-1} ; Z_{p}\right), Z_{p}\right) \text {. }
$$

Now, on the other hand, consider $\hat{Y}_{k-1}=Y_{k-1}^{(m)} \cup Y_{k-1}^{F}$, where $Y_{k-1}^{F}=\cup_{H \neq 0} Y_{k-1}^{H}$ $=\cup_{H \neq 0} X^{H}$. We claim that $\tilde{H}_{i}\left(\hat{Y}_{k-1} ; Z_{p}\right)=0$ for $i \neq m$. This amounts to showing that $H_{*}\left(\cup_{H \neq 0} X^{H} ; Z_{p}\right)=0$ for $*>m$. First of all, by induction on the rank of $G, r$, we may assume the Borel formula for any elementary abelian $p$-group of lesser rank. This induction implies that $n(H) \leqslant m$, for $H \neq 0$. Letting $S$ be any nonempty collection of nonzero subgroups of $G$ (without loss, not containing $G$ itself) one actually establishes the stronger fact that $H_{*}\left(\cup_{H \in S} X^{H} ; Z_{p}\right)=0$ if $*>m$. To show this one uses induction on the cardinality of $S$ and Mayer-Vietoris. Thus one is led to see it suffices to show that, for $M_{0} \in S$,

$$
H_{*}\left(X^{M_{0}}, \bigcup X^{M M_{0}} ; Z_{p}\right)=0, \text { for } *>n\left(M_{0}\right)
$$

where the union is over $S-\left\{M_{0}\right\}$. This latter fact is established by a double induction on rank $M_{0}$ and on the cardinality of $S-\left\{M_{0}\right\}$. 
There are two naturally arising cases. If there is some $M_{1}$ in $S-\left\{M_{0}\right\}$ with $n\left(M_{1} M_{0}\right)<n\left(M_{0}\right)$ one considers the homology sequence of the triple $\left(X^{M_{0}}, \cup X^{M M_{0}}, \cup X^{M M_{0}}\right.$ ), where the first union is over $S-\left\{M_{0}\right\}$ and the second is over $S-\left\{M_{0}, M_{1}\right\}$. An application of excision establishes the result in this case.

The second case is that every $M \in S$ has $n\left(M M_{0}\right)=n\left(M_{0}\right)$. Here, it suffices to show that if $M_{1}$ and $M_{2}$ both have $n\left(M_{1} M_{0}\right)=n\left(M_{2} M_{0}\right)=n\left(M_{0}\right)$ then $n\left(M_{0}\right)=$ $n\left(M_{1} M_{2} M_{0}\right)$ (by a Mayer-Vietoris argument). To demonstrate this last property use the fact that the Borel formula holds whenever a group of rank $\leqslant r-1$ is acting.

Now since $\tilde{H}_{i}\left(\hat{Y}_{k-1} ; Z_{p}\right)=0, i \neq m$, and the Borel formula holds on $\hat{Y}_{k-1}$, by Theorem $\mathrm{A}$ in the introduction (it is not hard to see that if $r>1, m-n(G)$ is always even if $p$ is odd)

$$
H_{m}\left(\hat{Y}_{k-1} ; Z_{p}\right)=Z_{p} \oplus F_{1},
$$

where $F_{1}$ is a free $Z_{p} G$ module.

Now consider $C_{*}\left(Y_{k-1}, \hat{Y}_{k-1} ; Z_{p}\right)$. We have the exact sequence,

$$
\begin{aligned}
0 & \rightarrow H_{k}\left(Y_{k-1} ; Z_{p}\right) \rightarrow C_{k}\left(Y_{k-1}, \hat{Y}_{k-1} ; Z_{p}\right) \rightarrow \ldots \\
& \rightarrow C_{m+1}\left(Y_{k-1}, \hat{Y}_{k-1} ; Z_{p}\right) \rightarrow H_{m}\left(\hat{Y}_{k-1} ; Z_{p}\right) .
\end{aligned}
$$

Since each $C_{i}\left(Y_{k-1}, \hat{Y}_{k-1} ; Z_{p}\right)$ is $Z_{p} G$ free, it follows that for $s \geqslant 2$,

$$
\operatorname{Ext}_{Z_{p} G}^{s+(k-m)}\left(H_{m}\left(\hat{Y}_{k-1} ; Z_{p}\right), Z_{p}\right)=\operatorname{Ext}_{Z_{p} G}^{s}\left(H_{k}\left(Y_{k-1} ; Z_{p}\right), Z_{p}\right) \text {. }
$$

Thus,

$$
\operatorname{Ext}_{Z_{p} G}^{s+(k-n)}\left(H_{n}\left(X ; Z_{p}\right), Z_{p}\right)=\operatorname{Ext}_{Z_{p} G}^{s+(k-m)}\left(H_{m}\left(\hat{Y}_{k-1} ; Z_{p}\right), Z_{p}\right)
$$

But since $H_{n}\left(X ; Z_{p}\right)$ and $H_{m}\left(\hat{Y}_{k-1} ; Z_{p}\right)$ are both stably $Z_{p}$, the result is that

$$
\operatorname{Ext}_{Z_{p} G}^{s+(k-n)}\left(Z_{p}, Z_{p}\right)=\operatorname{Ext}_{Z_{p} G}^{s+(k-m)}\left(Z_{p}, Z_{p}\right) \text {. }
$$

This implies that $n=m$, since $G$ has nonperiodic cohomology.

3. We now consider examples of Proposition 1 which are $n$-dimensional, $n-1$ connected.

Proposition 2. Suppose that $X$ satisfies the hypotheses of Proposition 1 and that $X$ is $n$-dimensional, $n-1$ connected. Then $H_{n}(X ; Z)=Z \oplus P$, where $P$ is $Z G$-projective and $Z$ has trivial $G$-action if $p$ is odd.

Proof. It was shown in [4] that there is a linear $G$-action on $S^{\boldsymbol{n}}$ and an equivariant map $\phi: X \rightarrow S^{n}$ having the properties described in the introduction. From the mapping cone $C_{\phi}$, we have the exact sequence

$$
0 \rightarrow H_{n+1}\left(C_{\phi} ; Z\right) \rightarrow H_{n}(X ; Z) \stackrel{\phi}{\rightarrow} H_{n}\left(S^{n} ; Z\right) \rightarrow H_{n}\left(C_{\phi} ; Z\right) \rightarrow 0 .
$$

Because $\phi_{*}$ is a $Z_{p}$-homology epimorphism we have

$$
0 \rightarrow H_{n+1}\left(C_{\phi} ; Z\right) \rightarrow H_{n}(X ; Z) \rightarrow \operatorname{Im} \phi_{*} \approx Z \quad \text { (with some action). }
$$

Also, $H_{n}\left(C_{\phi} ; Z\right)=Z_{k}$, where $(k, p)=1$ and $\operatorname{Im} \phi_{*}=(k)$. One may add free orbits of $n+1$ cells to $C_{\phi}$ to kill $H_{n}\left(C_{\phi} ; Z\right)$. This results in an $(n+1)$-dimensional, 
$n$-connected $G$-complex $Y$ such that $H_{n+1}(Y ; Z)=H_{n+1}\left(C_{\phi} ; Z\right) \oplus P, P$ a projective $Z G$ module. By [6, Lemma 3], $H_{n+1}(Y ; Z)$ is $Z G$-projective and then so is $H_{n+1}\left(C_{\phi} ; Z\right)$.

If $\operatorname{Im} \phi_{*} \approx Z$ has trivial $G$-action then one has

$$
\operatorname{Ext}_{Z G}\left(Z ; H_{n+1}\left(C_{\phi} ; Z\right)\right)=H^{1}\left(G ; H_{n+1}\left(C_{\phi} ; Z\right)\right)=0
$$

by $[6,4.11]$. Thus in this case, $(*)$ splits.

If $\operatorname{Im} \phi_{*} \approx Z$ has nontrivial $G$-action (denoted $Z^{-}$), it follows that $Z^{-} \otimes_{Z} Z^{-}$ $\approx Z$ has trivial (diagonal) $G$-action and that whenever $P$ is $Z G$-projective, so is $Z^{-} \otimes_{Z} P$ (diagonal action). This latter statement follows from the fact that $Z^{-} \otimes_{Z} Z G$ (diagonal action) is isomorphic, as $Z G$ modules, to $Z G$. Now tensoring (*) over $Z$ with $Z^{-}$one has the exact sequence of $Z G$ modules,

$$
0 \rightarrow H_{n+1}\left(C_{\phi} ; Z\right) \otimes_{Z} Z^{-} \rightarrow H_{n}(X ; Z) \otimes_{Z} Z^{-} \rightarrow Z \rightarrow 0 \text {. }
$$

By the argument above, $(*)^{\prime}$ splits. So,

$$
H_{n}(X ; Z) \otimes_{Z} Z^{-} \approx Z \oplus H_{n+1}\left(C_{\phi} ; Z\right) \otimes_{Z} Z^{-} .
$$

Now, tensoring again with $Z^{-}$over $Z$, one has $H_{n}(X ; Z) \approx Z^{-} \oplus H_{n+1}\left(C_{\phi} ; Z\right)$.

As an example, one may take the wedge of a standard linear action on $S^{n}$ with a bouquet of $n$-spheres permuted freely by $G$. On the other hand, suppose $Y$ is an $n$-dimensional $n-1$-connected $\mathrm{CW}$ complex with $G$-action such that $Y^{H}$ is $Z_{p}$ acyclic for each $H \triangleleft G, H \neq 0$. By [6, Lemma 3], $H_{n}(Y)$ is $Z G$-projective. Wedging $Y$ with a linear $S^{n}$ would yield other examples. However, it is a result of Swan (see [1, p. 178]) that $H_{n}(Y)$ must be stably free, when $G=Z_{p}$. Consequently, one can construct projective modules $P$ over $Z G, G=\left(Z_{p}\right)^{r}, r>1$, which could never be realised as an $H_{n}(Y)$, as above. Mainly, this is so because a necessary condition on $P$ is that the image of $P$ in $G_{0}(Z G) / S$ be zero, where $G_{0}(Z G)$ is the Grothendieck group on finitely generated $Z G$ modules and $S$ is the subgroup generated by signed permutation modules (see [1]). As a result it is not clear precisely when a projective $Z G$ module can be an $H_{n}(Y)$, as above. According to [1] the necessary condition above is sufficient for $G=Z_{p}$.

\section{BIBLIOGRAPHY}

1. J. E. Arnold, A solution of a problem of Steenrod for cyclic groups of prime order, Proc. Amer. Math. Soc. 62 (1977), 177-182.

2. A. Borel, Seminar on transformation groups, Ann. of Math. Studies, no. 46, Princeton Univ. Press, Princeton, N. J., 1960.

3. G. Bredon, Introduction to compact transformation groups, Academic Press, New York, 1972.

4. R. Dotzel, A converse of the Borel formula, Trans. Amer. Math. Soc. 250 (1979), 275-287.

5. L. Jones, The converse to the fixed point theorem of P. A. Smith. I, Ann. of Math. (2) 94 (1971), 52-68.

6. R. Oliver, Fixed point sets of group actions on finite acyclic complexes, Comment. Math. Helv. 50 (1975), 155-177.

7. D. S. Rim, Modules over finite groups, Ann. of Math. (2) 69 (1959), 700-712.

Department of Mathematics, University of TeXas, Austin, TeXas 78712 